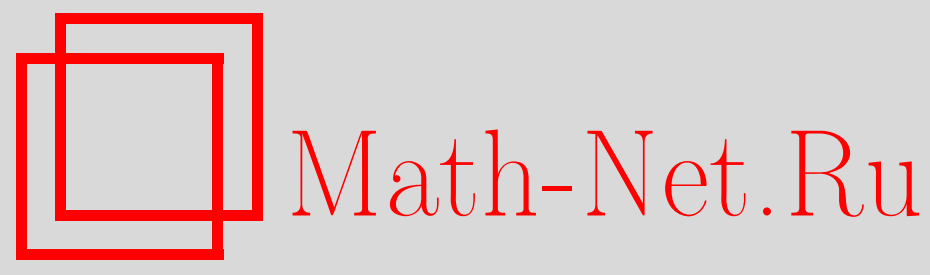

В. А. Колмыков, О неустойчивых ветвящихся графах, Дискрет. матем., 2012, том 24, выпуск 4, 81-90

DOI: https://doi.org/10.4213/dm1212

Использование Общероссийского математического портала Math-Net.Ru подразумевает, что вы прочитали и согласны с пользовательским соглашением http://www.mathnet.ru/rus/agreement

Параметры загрузки:

IP: 54.197 .130 .99

26 апреля 2023 г., 17:01:08 
УДК 519.17

\title{
О неустойчивых ветвящихся графах
}

\author{
() 2012 г. В. А. Колмыков
}

\begin{abstract}
Рассматриваются графы, получающиеся из деревьев заменой ребер другими графами, на которые накладываются некоторые условия. Исследуется множество всех неустойчивых неразложимых графов такого вида.
\end{abstract}

\section{1. Введение}

Углеводородной молекуле можно поставить в соответствие граф, вершинами которого являются атомы углерода, а ребрами - связи между атомами. Если этот граф двудольный, то наличие нуля в спектре его матрицы смежности интерпретируется как химическая неустойчивость молекулы (см. [1], §8.1, п. $1^{\circ}$ ). Поэтому граф называется неустойчивым, если в спектре его матрицы смежности есть нуль.

Молекула называется ветвящейся, если она состоит из каких-то фрагментов, соединенных друг с другом так, что при этом не возникает никаких циклов, кроме тех, которые имеются в самих фрагментах. Чтобы формализовать это понятие, можно воспользоваться следующей конструкцией. Рассмотрим дерево $T$, имеющее $r$ ребер, и $r$ других графов. Вместо каждого ребра дерева вклеим соответствующий граф. Полученный граф обозначим $T^{\varphi}$, где $\varphi$ - отображение, управляющее процессом вклейки. Всякий граф вида $T^{\varphi}$ называется ветвящимся над множеством вклеиваемых графов.

В $[2,3]$ рассматривалась подобная конструкция, когда дерево $T$ является цепью, а вклеиваемые графы изоморфны друг другу. При этом удалось получить полную классификацию случаев неустойчивости графа $T^{\varphi}$. В [4], в частности, исследовалась ситуация, когда $T=k_{1, n}$, а вклеиваемые графы - двудольные. При этом было показано, что при $n \geqslant 4$ неустойчивый граф $T^{\varphi}$ распадается на другие неустойчивые графы.

В настоящей статье рассматриваются произвольные деревья, а на вклеиваемые графы накладываются некоторые ограничения. Исследуется множество всех неустойчивых неразложимых ветвящихся графов такого вида.

Автор посвящает статью 75-летию своего учителя Ю. И. Манина.

\section{2. Основные понятия}

В настоящей статье мы рассматриваем конечные графы без петель и кратных ребер. Иными словами, граф - это пара $\left(V_{0}, V_{1}\right)$, где $V_{0}$ - конечное множество, а $V_{1}$ - некоторое множество, состоящее из двухэлементных подмножеств множества $V_{0}$. Для графа $G=\left(V_{0}, V_{1}\right)$ множества $V_{0}$ и $V_{1}$ иногда удобно обозначать соответственно $V_{0}(G)$ и $V_{1}(G)$. 
Через $\mathscr{G}$ обозначим множество всех графов (говоря о множествах графов, обычно предполагают, что их вершины принадлежат некоторому достаточно большому множеству $U$, причем изоморфные графы считаются равными). Через $\mathscr{B}$ обозначим подмножество в $\mathscr{G}$, состоящее из всех двудольных графов.

Непустой граф называется устойчивым, если в спектре его матрицы смежности нет нуля и неустойчивым в противном случае. Пустой граф emp $=(\varnothing, \varnothing)$ считается устойчивым.

Пример 1. Спектр матрицы смежности $n$-вершинной цепи $P_{n}$ состоит (см. [1], §2.6) из чисел $2 \cos (\pi k /(n+1))$, где $k \in\{1, \ldots, n\}$. Поэтому граф $P_{n}$ устойчив тогда и только тогда, когда $n$ четно.

Двукорневым графом с упорядоченными корнями называется граф, в котором отмечены две различные вершины, эти вершины называются корнями, причем один из корней считается первым, другой вторым. Множество всех двукорневых графов с упорядоченными корнями обозначается $\ddot{\mathscr{G}}$. Через $\ddot{\mathscr{B}}$ обозначим множество всех двудольных графов из $\ddot{\mathscr{G}}$. Пусть $G \in \ddot{\mathscr{G}}$. Первый корень графа $G$ обычно обозначают $\alpha(G)$, или просто $\alpha$, если ясно, о каком графе идет речь. Второй корень обозначают $\beta(G)$ (или просто $\beta$ ). Для $G \in \ddot{\mathscr{G}}$, через $G^{*}$ обозначим граф, получающийся из $G$ сменой порядка корней: $\alpha\left(G^{*}\right)=\beta(G)$, $\beta\left(G^{*}\right)=\alpha(G)$.

Ребро $\{u, v\} \in V_{1}(G)$ обозначается через $\overline{u v}$, а упорядоченная пара $(u, v)$ смежных вершин - через $\overrightarrow{u v}$. Множество всех упорядоченных пар смежных вершин будем обозначать $\overleftrightarrow{V}_{1}(G)$.

Пусть $G-$ некоторый граф. Отображение $\varphi: \overleftrightarrow{V}_{1}(G) \rightarrow \ddot{\mathscr{G}}$ назовем отображением вклейки, если

$$
\varphi(\overrightarrow{u v})=\varphi(\overrightarrow{v u})^{*}, \quad \overline{u v} \in V_{1}(G)
$$

Для каждого ребра $\overline{u v} \in V_{1}(G)$ проделаем следующее. Удалим это ребро (при удалении ребра все вершины графа сохраняются, исчезает лишь связь между концами ребра). Отождествим вершину $u$ с первым корнем графа $\varphi(\overrightarrow{u v})$, а вершину $v-$ с вторым корнем (однозначность гарантируется условием $\varphi(\overrightarrow{u v})=\varphi(\vec{v}) *)$. Полученный граф обозначим $G^{\varphi}$ и будем говорить, что графы $\varphi\left(\overleftrightarrow{V}_{1}(G)\right)$ вклеены в $G$.

Пример 2. При $n \geqslant 2$ через $\ddot{P}_{n}$ обозначим цепь $P_{n}$, в которой отмечены концевые вершины. В граф $K_{1,3}$ вклеим $\ddot{P}_{p}, \ddot{P}_{q}$ и $\ddot{P}_{r}$. Получим дерево, в котором одна вершина имеет степень 3 , три вершины имеют степень 1 , и $p+q+r-6$ вершин имеют степень 2. Это дерево называется трилистником и обозначается $T_{p, q, r}$.

Трилистник можно сконструировать и по-другому. В цепи $P_{p+q-1}$ первым корнем возьмем одну из концевых вершин, вторым корнем - вершину, находящуюся на расстоянии $p-1$ от первого корня. Полученный граф из $\ddot{G}$ обозначим $F$.

Пусть $G=P_{3}$ и $V_{0}(G)=\{u, v, w\}$, где $u$ и $w-$ концевые вершины. Положим

$$
\varphi(\overrightarrow{u v})=F, \quad \varphi(\overrightarrow{v u})=F^{*}, \quad \varphi(\overrightarrow{v w})=\ddot{P}_{r}, \quad \varphi(\overrightarrow{w v})=\ddot{P}_{r}^{*} .
$$

Тогда $G^{\varphi}=T_{p, q, r}$. Трилистники используются в исследованиях алгебр Ли и теории представлений (см., например, [5], гл. VI, §4; [6], гл. 4; [7]; [8]).

Пусть $M \subseteq \ddot{G}$. Граф назовем ветвящимся над $M$, если он имеет вид $T^{\varphi}$, где $T-$ дерево, отличное от $P_{1}$, а $\varphi: \overleftrightarrow{V}_{1}(T) \rightarrow \mathcal{M}$ есть отображение вклейки. 
Говорят, что граф $G$ получается из графов $F$ и $H$ склейкой по вершине, если в $F$ и $H$ можно выбрать по одной вершине так, что $G$ получается из $F$ и $H$ отождествлением выбранных вершин.

Пусть $\mathscr{H}$ - некоторое множество графов. Граф $G$ называется разложимым над $\mathscr{H}$, если его можно получить из графов $H_{1}, H_{2} \in \mathcal{H}$ склейкой по вершине, причем $H_{1} \neq G \neq H_{2}$. Например, блок (то есть, связный граф, не имеющий разделяющих вершин) не является разложимым.

Подмножество в $\mathscr{H}$, состоящее из всех графов, неразложимых над $\mathscr{H}$, назовем базой множества $\mathscr{H}$. В [9] найдена база множества всех неустойчивых деревьев. В настоящей статье получены более общие утверждения.

\section{3. Формулировка результатов}

Для графа $G \in \ddot{\mathscr{G}}$ через $G_{\alpha}$ (соответственно, $G_{\beta}$; соответственно, $G_{\alpha \beta}$ ) обозначается граф, получающийся из $G$ удалением первого корня (соответственно, второго корня; соответственно, обоих корней).

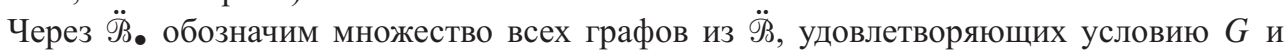
таких, что $G_{\alpha \beta}$ устойчивы, или $G_{\alpha}$ и $G_{\beta}$ устойчивы.

Пример 3. Используя обозначения из примера 2 и результат из примера 1 , находим, что $\ddot{P}_{n} \in \ddot{\mathscr{B}}_{\bullet}$.

Интересно заметить, что на ̈̈̊ существует естественная структура полугруппы (см. лемму 2).

Ребро дерева называется концевым, если степень одной из его вершин равна 1. Концевое ребро назовем периферийным, если степень одной из его вершин больше 1.

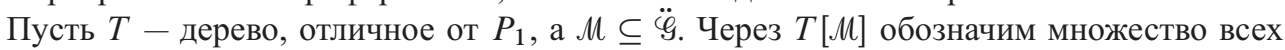
графов вида $T^{\varphi}$, где $\varphi: \overleftrightarrow{V}_{1}(T) \rightarrow \mathcal{M}$ - такое отображение вклейки, что граф $\varphi(\overrightarrow{u v})$ устойчив тогда и только тогда, когда ребро $\overline{u v}$ является периферийным.

Положим

$$
\Upsilon[M]=\bigcup_{n, p, q, r}\left(P_{n}[\mathcal{M}] \cup T_{p, q, r}[\mathcal{M}]\right) \text {. }
$$

В следующем разделе будет показано, что графы из $\Upsilon[\mathcal{M}]$ неустойчивы.

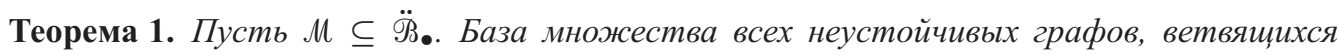
над $\mathcal{M}$ является подмножеством в $\Upsilon$ я

Замечание 1. В лемме 6 будет показано, что эта база является собственным подмножеством в $\Upsilon[\mathcal{M}]$ для многих $\mathcal{M}$.

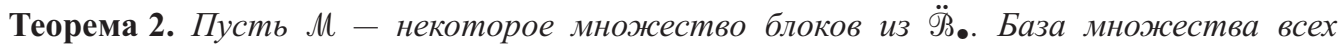
неустойчивых графов, ветвящихся над $\mathcal{M}$, совпадает с $[$ [M].

Замечание 2. Для элементов множества $\Upsilon[\mathcal{M}] \backslash P_{2}[\mathcal{M}]$ имеется некоторая аналогия с адронами. Мезон можно представлять как трубку цветового потока с кварками, прикрепленными на концах (см. [10], с. 183). Элемент вида $P_{n}[\mathcal{M}]$ - это цепь, в которой внутренние ребра заменены неустойчивыми графами, а концевые - устойчивыми. В структуру бариона входят три кварка. Элемент вида $T_{p, q, r}[\mathcal{M}]$ - это трилистник, в котором внутренние ребра заменены неустойчивыми графами, а концевые - устойчивыми. 


\section{4. Доказательства}

Характеристический многочлен

$$
G(\lambda)=\left|\lambda I-A_{G}\right|
$$

матрицы смежности $A_{G}$ непустого графа $G$ не зависит от нумерации вершин, он называется характеристическим многочленом графа. Если $G-$ пустой граф, то $G(\lambda)=1$.

В спектральной теории графов хорошо известна (см., например, [1], §6.1), независимо доказанная многими авторами, теорема о разрезании по вершине. Приведем ее в удобной для нас формулировке.

Пусть граф $H$ можно представить в виде склейки двух графов $X$ и $Y$ посредством отождествления некоторой вершины $x$ графа $X$ и некоторой вершины $y$ графа $Y$. Теорема о разрезании по вершине утверждает, что

$$
H(\lambda)=X_{x}(\lambda) Y(\lambda)+X(\lambda) Y_{y}(\lambda)-\lambda X_{x}(\lambda) Y_{y}(\lambda)
$$

Приведем несколько простейших следствий этой теоремы.

Пусть $H^{(1)}, \ldots, H^{(k)}$ - некоторые графы. В каждом графе $H^{(i)}$ отметим некоторую вершину $u_{i}$. Через $H$ обозначим граф, получающийся из $H^{(1)}, \ldots, H^{(k)}$ отождествлением вершин $u_{1}, \ldots, u_{k}$. Тогда

$$
H(0)=\sum_{i}\left(H^{(i)}(0) \prod_{j \neq i} H_{u_{j}}^{(j)}(0)\right) .
$$

На множестве $\ddot{\mathscr{G}}$ определим ассоциативную операцию следующим образом: $F \bullet G$ получается из $F$ и $G$ отождествлением $\beta(F)$ и $\alpha(G)$, причем полагаем по определению

$$
\alpha(F \bullet G)=\alpha(F), \quad \beta(F \bullet G)=\beta(G) .
$$

Из теоремы о разрезании по вершине очевидно следует, что

$$
\begin{aligned}
(F \bullet G)(0) & =F_{\beta}(0) G(0)+F(0) G_{\alpha}(0), \\
(F \bullet G)_{\alpha \beta}(0) & =F_{\alpha \beta}(0) G_{\beta}(0)+F_{\alpha}(0) G_{\alpha \beta}(0), \\
(F \bullet G)_{\alpha}(0) & =F_{\alpha \beta}(0) G(0)+F_{\alpha}(0) G_{\alpha}(0), \\
(F \bullet G)_{\beta}(0) & =F_{\beta}(0) G_{\beta}(0)+F(0) G_{\alpha \beta}(0) .
\end{aligned}
$$

Лемма 1. Справедливы следующие утверждения.

(1) Если граф $G \in \ddot{\mathscr{乃}}$ устойчив, то графы $G_{\alpha} u G_{\beta}$ неустойчивы.

(2) Если графы $G, H \in \ddot{\Re}$ устойчивы, то граф $G \bullet H$ неустойчив.

(3) Если $G \in \ddot{\mathscr{乃}}$, то условие устойчивости обоих графов $G$ и $G_{\alpha \beta}$ несовместно с условием устойчивости обоих графов $G_{\alpha} u G_{\beta}$.

(4) Пусть $F \in \ddot{\Re}_{.}$. Если граф $F$ устойчив, то $F_{\alpha \beta}$ устойчив, а $F_{\alpha}$ и $F_{\beta}$ неустойчивы. Если граф $F$ неустойчив, то $F_{\alpha \beta}$ неустойчив, а $F_{\alpha}$ и $F_{\beta}$ устойчивы.

(5) Графы из $K_{1, n}[\ddot{\mathscr{B}}]$ неустойчивы для любого натурального $n$. 
(6) Если граф $G$ можно склеить из неустойчивых графов $F$ и $H$, то $G$ неустойчив.

Доказательство. Необходимым условием устойчивости двудольного графа является четность числа его вершин (см. изложение после следствия теоремы 8.2 в [1]). Из этого очевидно следуют утверждения 1-3.

Утверждение 4 следует из утверждений 1 и 3.

Докажем утверждение 5 . В графе $K_{1,1}$ нет периферийных ребер, поэтому всякий граф из $K_{1,1}[\ddot{\Re}]$ неустойчив.

Пусть $n \geqslant 2$. Возьмем $H \in K_{1, n}[\ddot{\Re}]$. Существуют устойчивые двудольные графы $H^{(1)}, \ldots, H^{(k)}$, обладающие следующим свойством: в каждом графе $H^{(i)}$ можно выделить некоторую вершину $u_{i}$ так, что $H$ получается из $H^{(1)}, \ldots, H^{(k)}$ отождествлением вершин $u_{1}, \ldots, u_{k}$. Применяя формулу (1) и утверждение 1 леммы, получаем, что $H(0)=0$.

Утверждение 6 следует из теоремы о разрезании по вершине.

Определим отображение $s: \ddot{\mathscr{Я}} \bullet \rightarrow \mathbf{Z}_{2}$ следующим образом:

$$
s(G)=\overline{0} \Longleftrightarrow G(0)=0 .
$$

Лемма 2. Пара $\left(\ddot{\Re}_{\bullet}, \bullet\right)$ есть полугруппа, а отображение $s:\left(\ddot{\Re}_{\bullet}, \bullet\right) \rightarrow\left(\mathbf{Z}_{2},+\right)$ есть гомоморфизм.

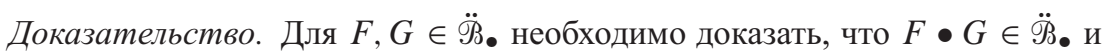

$$
s(F \bullet G)=s(F)+s(G) .
$$

1. Пусть графы $F$ и $G$ неустойчивы. По лемме 1 , графы $F_{\alpha}, F_{\beta}, G_{\alpha}, G_{\beta}$ устойчивы. Используя (3), получаем, что

$$
\begin{aligned}
& (F \bullet G)_{\alpha}(0)=F_{\alpha}(0) G_{\alpha}(0) \neq 0, \\
& (F \bullet G)_{\beta}(0)=F_{\beta}(0) G_{\beta}(0) \neq 0,
\end{aligned}
$$

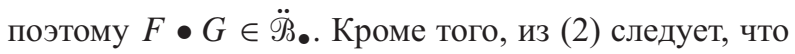

$$
(F \bullet G)(0)=0,
$$

поэтому

$$
s(F \bullet G)=\overline{0}=s(F)+s(G) .
$$

2. Пусть графы $F$ и $G$ устойчивы. По лемме 1 , графы $F_{\alpha}, F_{\beta}, G_{\alpha}, G_{\beta}, F \bullet G$ неустойчивы, а $F_{\alpha \beta}$ и $G_{\alpha \beta}$ устойчивы. Используя (3), получаем, что

$$
\begin{aligned}
& (F \bullet G)_{\alpha}(0)=F_{\alpha \beta}(0) G(0) \neq 0, \\
& (F \bullet G)_{\beta}(0)=F(0) G_{\alpha \beta}(0) \neq 0,
\end{aligned}
$$

поэтому $F \bullet G \in \ddot{\Re}_{\bullet}$. Кроме того,

$$
s(F \bullet G)=\overline{0}=s(F)+s(G) .
$$


3. Пусть граф $F$ неустойчив, а $G$ устойчив. По лемме 1 , графы $F_{\alpha}, F_{\beta}, G_{\alpha \beta}$ устойчивы, а $F_{\alpha \beta}, G_{\alpha}, G_{\beta}$ неустойчивы. Используя (2), получаем, что

$$
\begin{aligned}
(F \bullet G)(0) & =F_{\beta}(0) G(0) \neq 0, \\
(F \bullet G)_{\alpha \beta}(0) & =F_{\alpha}(0) G_{\alpha \beta}(0) \neq 0 .
\end{aligned}
$$

Поэтому $F \bullet G \in \ddot{\Re}$. Кроме того, из (2) следует, что

$$
(F \bullet G)(0)=F_{\beta}(0) G(0) \neq 0,
$$

поэтому

$$
s(F \bullet G)=\overline{1}=s(F)+s(G) .
$$

4. Случай, когда граф $F$ устойчив, а $G$ неустойчив, рассматривается аналогично предыдущему.

Лемма 3. Пусть $G_{1}, \ldots, G_{m} \in \ddot{\Re}_{\bullet}$, где $m \geqslant 1$. Граф $G_{1} \bullet \ldots \bullet G_{m}$ устойчив тогда $и$ только тогда, когда среди графов $G_{1}, \ldots, G_{m}$ есть нечетное число устойчивых.

Доказательство. Из леммы 2 следует, что условие

$$
s\left(G_{1} \bullet \ldots \bullet G_{m}\right)=\overline{1}
$$

равносильно равенству

$$
s\left(G_{1}\right)+\ldots+s\left(G_{m}\right)=\overline{1} .
$$

Через $\ddot{\mathscr{P}}_{*}$ обозначим множество всех устойчивых графов из $\ddot{\Re}$. Степень вершины $u$ в графе $G$ обозначим $\operatorname{deg}_{G} u$.

Лемма 4. Для любого дерева $T$, графы из $T\left[\ddot{\Re}_{\bullet} \cup \ddot{\Re}_{*}\right]$ неустойчивы.

Доказательство. Доказательство проведем индукцией по числу $n$ вершин дерева. При $n \leqslant 3$ справедливость леммы следует из части 5 леммы 1.

Пусть утверждение верно для $n \leqslant k$, и пусть

$$
\left|V_{0}(T)\right|=k+1 .
$$

Если $T=K_{1, k}$, то справедливость леммы следует из части 5 леммы 1.

Пусть $T \neq K_{1, k}$. Вершина степени 1 назовем концевой. Предконцевой вершиной назовем вершину, инцидентную хотя бы одной концевой, и ровно одной не концевой. Так как $T \neq K_{1, k}$, в $T$ есть предконцевая вершина. Обозначим ее $u$. Пусть $u_{0}-$ неконцевая вершина, инцидентная $u$, а $u_{1}, \ldots, u_{s}$ - концевые вершины, инцидентные $u$.

Через $F$ обозначим дерево, получающееся из $T$ удалением вершин $u_{1}, \ldots, u_{s}$. Дерево $T$ получим отождествлением вершины $u$ дерева $F$ и вершины степени $s$ дерева $K_{1, s}$. Отметим, что

$$
\operatorname{deg}_{T} u=s+1 \geqslant 2, \quad \operatorname{deg}_{F} u=1, \quad \operatorname{deg}_{T} u_{0}=\operatorname{deg}_{F} u_{0} \geqslant 2 .
$$

Рассмотрим граф $G=T^{\varphi} \in T\left[\ddot{\Re}_{\bullet} \cup \ddot{乃}_{*}\right]$.

Если $s \geqslant 2$, то $G$ можно представить в виде склейки некоторого графа $X$ и некоторого графа $Y \in K_{1, s}\left[\ddot{\Re}_{\bullet} \cup \ddot{\Re}_{*}\right]$ посредством отождествления некоторой вершины $x$ графа $X$ 
и той вершины $y$ графа $Y$, которая соответствует вершине степени $s$ графа $K_{1, s}$. Из части 5 (соответственно, части 1) леммы 1 следует, что граф $Y$ (соответственно, граф $Y_{y}$ ) неустойчив. Тогда из теоремы о разрезании по вершине следует, что

$$
G(0)=X_{x}(0) Y(0)+X(0) Y_{y}(0)=0 .
$$

Пусть $s=1$. Положим $X=F^{\psi}$, где $\psi=\varphi \mid \overleftrightarrow{V}_{1}(F)$. Тогда $G$ можно представить в виде склейки графа $X$ и некоторого устойчивого графа $Y$ посредством отождествления вершины $u$ графа $X$ и некоторой вершины $y$ графа $Y$. По лемме 1 , граф $Y_{y}$ неустойчив.

Покажем, что $X_{u} \in F\left[\ddot{\mathscr{B}}_{\bullet} \cup \ddot{\mathscr{B}}_{*}\right]$. Ребро $\overline{u_{0} u}$ является непериферийным в $T$, поэтому граф $\psi\left(\overrightarrow{u_{0} \vec{u}}\right)$ неустойчив. Значит, $\psi\left(\overrightarrow{u_{0} \vec{u}}\right)_{\beta}$ устойчив. Поэтому в нем имеется не менее двух вершин. Отметим некоторую вершину, отличную от первого корня, и будем считать ее вторым корнем. Полученный граф из $\ddot{\mathscr{B}}_{*}$ обозначим $Z$.

Ребро $\overline{u_{0} u}$ является периферийным в $F$. Ребро дерева $F$, отличное от $\overline{u_{0} u}$, является периферийным в $F$ тогда, и только тогда, когда оно является периферийным в $T$. Поэтому $X=F^{\xi}$, где $\xi$ отличается от $\psi$ лишь значениями на ${\overrightarrow{u_{0}}}_{\vec{u}}$ и $\overrightarrow{u \vec{u}} 0$, а именно:

$$
\xi\left(\overrightarrow{u_{0} \vec{u}}\right)=Z, \quad \xi\left(\vec{u}_{0}\right)=Z^{*} .
$$

Итак, $X_{u} \in F\left[\ddot{\mathscr{\Re}}_{\bullet} \cup \ddot{\mathscr{\Re}}_{*}\right]$. Тогда, по предположению индукции, граф $X_{u}$ неустойчив. Теперь из части 6 леммы 1 следует, что граф $G$ неустойчив.

В остальной части статьи неразложимость (соответственно, разложимость) означает неразложимость (соответственно, разложимость) над множеством всех неустойчивых графов, ветвящихся над $\mathcal{M}$. Каждый раз из контекста будет ясно, о каком $\mathcal{M}$ идет речь.

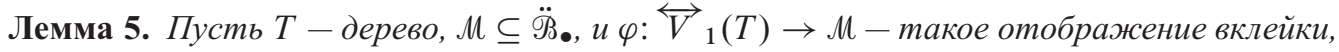
что граф $T^{\varphi}$ является неустойчивым и неразложимым. Тогда $T^{\varphi} \in T[M]$.

Доказательство. Докажем, что для устойчивости графа $\varphi(\overrightarrow{u v})$ необходимо и достаточно, чтобы ребро $\overline{u v}$ было периферийным.

Необходимость. Предположим противное: именно, пусть граф $\varphi(\overrightarrow{u v})$ устойчив для некоторого непериферийного ребра $\overline{u v}$ дерева $T$. Положим $G=\varphi(\overrightarrow{u v})$. Так как ребро $\overline{u v}$ является непериферийным, существуют такие ветвящиеся над $M$ графы $F, H \in \ddot{\mathscr{B}}$, что

$$
T^{\varphi} \simeq F \bullet G \bullet H .
$$

Пусть графы $F$ и $H$ являются неустойчивыми. Используя (2)-(3), получаем, что

$$
\begin{aligned}
(F \bullet G \bullet H)(0) & =(F \bullet G)_{\beta}(0) H(0)+(F \bullet G)(0) H_{\alpha}(0) \\
& =\left(F_{\beta}(0) G(0)+F(0) G_{\alpha}(0)\right) H_{\alpha}(0) \\
& =F_{\beta}(0) G(0) H_{\alpha}(0)
\end{aligned}
$$

Так как $F \bullet G \bullet H$ является неустойчивым, $F_{\beta}$ или $H_{\alpha}$ (пусть для определенности, первый из них) неустойчив. Используя (2), получаем, что

$$
(F \bullet G)(0)=F_{\beta}(0) G(0)+F(0) G_{\alpha}(0)=0 .
$$

Итак, граф $F \bullet G$ является неустойчивым, поэтому $F \bullet G \bullet h$ является разложимым. Это противоречит неразложимости $T^{\varphi}$. 
Пусть теперь графы $F$ и $H$ являются устойчивыми. По лемме 1 , графы $F_{\beta}$ и $H_{\alpha}$ являются неустойчивыми, а граф $G_{\alpha \beta}$ является устойчивым. Используя (2)-(3), получаем, что

$$
\begin{aligned}
(F \bullet G \bullet H)(0) & =(F \bullet G)_{\beta}(0) H(0)+(F \bullet G)(0) H_{\alpha}(0) \\
& =\left(F_{\beta}(0) G_{\beta}(0)+F(0) G_{\alpha \beta}(0)\right) H(0) \\
& =F(0) G_{\alpha \beta}(0) H(0) \neq 0,
\end{aligned}
$$

что противоречит неустойчивости $T^{\varphi}$.

Пусть теперь $F$ является устойчивым, а $H$ является неустойчивым. По лемме 1 , граф $F \bullet G$ является неустойчивым, поэтому $F \bullet G \bullet h$ является разложимым. Это противоречит неразложимости $T^{\varphi}$.

Случай, когда $F$ является неустойчивым, а $H$ является устойчивым, рассматривается аналогично предыдущему случаю.

Достаточность. Предположим противное: именно, пусть граф $\varphi(\overrightarrow{u v})$ является неустойчивым для некоторого периферийного ребра $\overline{u v}$ дерева $T$. Положим $G=\varphi(\overrightarrow{u v})$. Так как ребро $\overline{u v}$ является периферийным, существует ветвящийся над $\mathcal{M}$ граф $F \in \ddot{\mathscr{B}}$ такой, что

$$
T^{\varphi} \simeq F \bullet G .
$$

Если граф $F$ является неустойчивым, то $F \bullet G$ является разложимым. Это противоречит неразложимости $T^{\varphi}$.

Пусть граф $F$ является устойчивым. По лемме 1 , граф $G_{\alpha}$ является устойчивым. Используя (2), получаем, что

$$
(F \bullet G)(0)=F_{\beta}(0) G(0)+F(0) G_{\alpha}(0)=F(0) G_{\alpha}(0) \neq 0,
$$

что противоречит неустойчивости графа $T^{\varphi}$.

Доказательство теоремы 1 . Пусть $T$ - дерево, $\varphi: \overleftrightarrow{V}_{1}(T) \rightarrow \mathcal{M}$ - такое отображение вклейки, что граф $T^{\varphi}$ является неустойчивым и неразложимым. Покажем, что $T^{\varphi} \in \Upsilon[\mathcal{M}]$.

Из леммы 5 следует, что $T^{\varphi} \in T[M]$. Остается доказать, что $T=P_{n}$ или $T=T_{p, q, r}$.

Покажем, что в $T$ нет двух вершин степени, большей или равной 3 . Действительно, в противном случае существуют такие деревья $T_{1}, T_{2} \in \ddot{\mathscr{B}}$, что

$$
T=T_{1} \bullet \ddot{P}_{k} \bullet T_{2}, \quad \operatorname{deg} \beta\left(T_{1}\right) \geqslant 2, \quad \operatorname{deg} \alpha\left(T_{2}\right) \geqslant 2 .
$$

Положим

$$
\varphi_{i}=\left.\varphi\right|_{\overleftrightarrow{V}_{1}\left(T_{i}\right)}, \quad \psi=\left.\varphi\right|_{\mathbb{V}_{1}\left(\ddot{P}_{k}\right)}
$$

Если в графах $T_{1}^{\varphi_{1}}, \ddot{P}_{k}^{\psi}$ и $T_{2}^{\varphi_{2}}$ сохранить отмеченность вершин, унаследованных от $T_{1}$, $\ddot{P}_{k}$ и $T_{2}$ соответственно, то получаем, что

$$
T^{\varphi}=T_{1}^{\varphi_{1}} \bullet \ddot{P}_{k}^{\psi} \bullet T_{2}^{\varphi_{2}} .
$$

Ребро является периферийным в $T$ тогда и только тогда, когда оно является периферийным в $T_{1}$ или $T_{2}$. Поэтому $T_{i}^{\varphi_{i}} \in T_{i}[\mathcal{M}]$. По лемме 4 , граф $T_{i}^{\varphi_{i}}$ является неустойчивым. 
Никакое ребро цепи $\ddot{P}_{k}$ не является периферийным в $T$. Поэтому граф $\psi(\overrightarrow{u v})$ является неустойчивым для любого ребра $\overline{u v}$. Значит,

$$
\ddot{P}_{k}^{\psi}=G_{1} \bullet \ldots \bullet G_{k-1}
$$

для некоторых неустойчивых графов $G_{1}, \ldots G_{k-1} \in \ddot{\Re}$. По лемме 3 , граф $\ddot{P}_{k}^{\psi}$ является неустойчивым. Из части 6 леммы 1 следует, что и $T_{1}^{\varphi_{1}} \bullet \ddot{P}_{k}^{\varphi}$ является неустойчивым. Значит, $T^{\varphi}$ является разложимым. Тем самым получено противоречие.

Покажем, что в $T$ нет вершины степени, большей или равной 4. Действительно, в противном случае существуют деревья $T_{1}$ и $T_{2}$ такие, что $T$ можно получить, отождествляя некоторую вершину $u_{1} \in V_{0}\left(T_{1}\right)$ с некоторой вершиной $u_{2} \in V_{0}\left(T_{2}\right)$, причем $\operatorname{deg} u_{i} \geqslant 2$. Положим

$$
\varphi_{i}=\varphi \mid \overleftrightarrow{V}_{1}\left(T_{i}\right)
$$

Граф $T^{\varphi}$ можно получить из $T_{1}^{\varphi_{1}}$ и $T_{2}^{\varphi_{2}}$, отождествляя вершины $u_{1}$ и $u_{2}$.

Ребро является периферийным в $T$ тогда и только тогда, когда оно является периферийным в $T_{1}$ или $T_{2}$. Поэтому $T_{i}^{\varphi_{i}} \in T_{i}[\mathcal{M}]$. По лемме 4 , граф $T_{i}^{\varphi_{i}}$ является неустойчивым. Значит, $T^{\varphi}$ является разложимым. Тем самым получено противоречие.

Отметим, что отсутствие вершин степени, большей или равной 4 , легко вывести из результатов [4], но представляется затруднительным привести нужный результат из-за существенного различия в терминологии.

Доказав теорему 1 , хотелось бы убедиться в том, что в некоторых случаях база является собственным подмножеством в $\Upsilon[\mathcal{M}]$. Это будет показано в лемме 6.

На множестве $\ddot{\mathscr{G}}$ определим неассоциативную операцию следующим образом: $F \circ G$ получается из $F$ и $G$ отождествлением $\beta(F)$ и $\alpha(G)$, причем полагаем по определению

$$
\alpha(F \circ G)=\alpha(F), \quad \beta(F \circ G)=\beta(F) .
$$

Лемма 6. Справедливы следующие утверждения.

(1) Если $F, G \in \ddot{\mathscr{乃}}_{\bullet}$, причем $F$ является устойчивым, а $G$ является неустойчивым, то $F \circ G \in \ddot{\mathscr{B}}_{\bullet}$.

(2) Пусть $F, G \in \ddot{\Re}_{\bullet}$, причем $F$ является устойчивым, а $G$ является неустойчивым. Если $\{F, G, F \circ G\} \subseteq M \subseteq \ddot{\mathscr{乃}}_{\bullet}$, то база множества всех неустойчивых графов, ветвящихся над $\mathcal{M}$, является собственным подмножеством в $\Upsilon[\mathcal{M}]$.

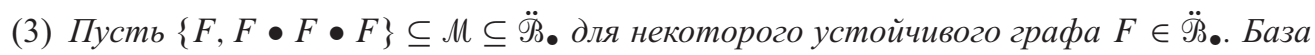
множества всех неустойчивых графов, ветвящихся над М, является собственным подмножеством в $[$ М].

Доказательство. Докажем первое утверждение. По лемме 3 , граф $F \bullet G$ является устойчивым. Поэтому устойчивым является и $F \circ G$. Граф $(F \circ G)_{\alpha \beta}$ является объединением графов $F_{\alpha \beta}$ и $G_{\alpha}$, которые устойчивы (по лемме 1$)$. Поэтому $(F \circ G)_{\alpha \beta}$ тоже является устойчивым. Значит, $F \circ G \in \ddot{\Re}_{\text {•. }}$

Докажем второе утверждение. В предыдущей части доказательства мы видели, что граф $F \circ G$ является устойчивым. Поэтому $F \bullet(F \circ G) \in P_{3}[M] \subseteq \Upsilon[M]$. Граф $F \bullet(F \circ G)$ получен из $F \bullet F$ и $G$ склейкой по вершине. По лемме 3 , граф $F \bullet F$ является неустойчивым. Значит, граф $F \bullet(F \circ G)$ является разложимым. 
Докажем третье утверждение. По лемме 3 , граф $F \bullet F$ является неустойчивым, а $F \bullet F \bullet F$ является устойчивым. Граф $F \bullet(F \bullet F \bullet F) \in P_{3}[M] \subseteq \Upsilon[\mathcal{M}]$ является разложимым, так как он получен из графов $F \bullet F$ и $F \bullet F$ склейкой по вершине.

Доказательство теоремы 2. По теореме 1, база является подмножеством в $[$ М]. Из леммы 4 следует, что все графы из $\Upsilon$ [M] являются неустойчивыми. Остается доказать, что все они являются неразложимыми.

Пусть $G \in P_{n}[\mathcal{M}]$. Если $n=2$, то граф $G$ неразложим, так как он является блоком.

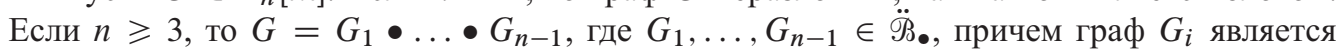
устойчивым тогда и только тогда, когда $i \in\{1, m\}$. Предположим, что $G$ является разложимым: $G=F \bullet H$, где $F$ и $H$ являются неустойчивыми. Так как графы $G_{i}$ являются блоками, справедливо представление $F=G_{1} \bullet \ldots \bullet G_{k}$, где $k \neq n-1$. По лемме 3, граф $F$ является устойчивым. Тем самым получено противоречие.

Пусть $G \in T_{p, q, r}[M]$. Предположим, что $G$ является разложимым, то есть представим в виде склейки неустойчивых графов $F$ и $H$. Так как все графы, вклеенные в $T_{p, q, r}$, являются блоками, по крайней мере один из графов $F$ или $H$ можно представить в виде $G_{1} \bullet \ldots \bullet G_{k}$, где $k \geqslant 1, G_{i} \in \ddot{\mathscr{乃}}_{\bullet}$ для всех $i$, и ровно один из графов $G_{i}$ является устойчивым, в то время как все остальные являются неустойчивыми. По лемме 3 , граф $G_{1} \bullet \ldots \bullet G_{k}$ является устойчивым. Тем самым получено противоречие.

\section{Список литературы}

1. Цветкович Д., Дуб М., Захс Х., Спектры графов. Теория и применение. Наукова думка, Киев, 1984.

2. Колмыков В. А., К исследованию устойчивости периодических углеводородных молекул. $\mathrm{Cu-}$ бирский журн. индустр. матем. (2006) 9, №1, 85-90.

3. Колмыков В. А., К исследованию устойчивости углеводородных молекул: пульсации нуля в спектрах периодических графов. Сибирский журн. индустр. матем. (2007) 10, №1, 90-95.

4. Колмыков В. А., Моноиды и распад. Сибирский матем. журн. (2007) 48, №6, 1246-1249.

5. Бурбаки Н., Группы и алгебры Ли. Группы Кокстера и системы Титса. Группы, порожденные отражениями. Системы корней. Мир, Москва, 1972.

6. Кац В., Бесконечномерные алгебры Ли. Мир, Москва, 1993.

7. Бернштейн И. Н., Гельфанд И. М., Пономарев В. А., Функторы Кокстера и теорема Габриеля. Успехи матем. наук (1973) 28, №2, 19-33.

8. Колмыков В. А., Нулевой конус формы Картана-Титса и графы Дынкина. В сб.: Сборник трудов семинара под руководством И. Р. Шафаревича, 2. Фонд математического образования и просвещения, Москва, 2000, с. 3-14.

9. Колмыков В. А., Об устойчивых и неустойчивых деревьях. Дискретная математика (2005) 17, №2, 150-152.

10. Манин Ю. И., Струны. В сб.: Элементарные частицы. (Кобзарев И. Ю., Манин Ю. И., ред.). ФАЗИС, Москва, 2000, с. 181-197. 